\title{
The nucleus locus coeruleus modulatory effect on memory formation: A literature review
}

\author{
Behrooz Khakpour-Taleghani 1,2,*, Mohammad Akhoondian², Adele Jafari²,3 \\ ${ }^{1}$ Neuroscience Research Center, School of Medicine, Guilan University of Medical Sciences, Guilan, Iran \\ 2Department of Physiology, School of Medicine, Guilan University of Medical Sciences, Rasht, Iran \\ 3 Cellular and Molecular Research Center, School of Medicine, Guilan University of Medical Sciences, Rasht, Iran
}

\begin{abstract}
The nucleus locus coeruleus (LC), the main source of norepinephrine in the brain, is connected to memory processing regions such as the hippocampus and baso-lateral amygdala (BLA). The LC and its mostly associated noradrenergic projections, play an important role in memory formation parallel to other neurotransmitter systems. It has been suggested that the unique response characteristics of LC to various situations strengthens different memories formation. Here, we review key related findings of LC effect on memory (avoidance, spatial, cognitive) formation, memory processing regions, memory molecular mechanisms as well as its role in memory related disorders. Literature review was conducted by extensive search on ISI, PubMed and Scopus, online databases from May 2021 to July 2021. According to the obtained results, LC noradrenergic projections to memory processing areas of the brain, can modulate the encoding, consolidation, and retrieval for different memory types. Also, the $\mathrm{LC}$ regulates neurogenesis and neural plasticity in different areas of the brain. Evidences suggested that dysfunction of the LC and its associated noradrenergic system may lead to cognitive impairment or a variety of memory-related disorders, including Alzheimer's disease. Finally, it can be concluded that the locus coeruleus noradrenergic system may be a suitable target for the treatment of different memory/cognitive disorders.
\end{abstract}

Keywords: Locus coeruleus, Memory, Neurogenesis, Neuronal plasticity, Alzheimer's disease

\section{Introduction}

Memory is the dynamic, unlimited process that information is learned, stored, and retrieved through it [1]. This complex brain function has various types, such as avoidance, spatial and recognition memory and each involves different brain areas [2]. Memory formation comes from interactions between different cells, neurons, neural circuits, and nuclei within the brain [3, 4]. The complicated neurobiological interplay between different parts causes molecular changes in neurons and non-neuronal cells, formation of new

\section{"Corresponding author:}

Behrooz Khakpour-Taleghani, Ph.D

Department of Physiology, School of Medicine,

Guilan University of Medical Sciences, Guilan, Iran

Tel/Fax: +98 1333690006

Email: khakpour_b@yahoo.com

https://orcid.org/oooo-0002-8192-0188

Received: August, 31, 2021

Accepted: September, 25, 2021 memories, and finally leads to individual behavioral strategies and decisions alteration [4].

Although memory formation is a result of the interactions between different chemical factors and brain cells, given its central role in inducing cellular and circuit changes, norepinephrine (NE) has great importance in memory formation [5]. The nucleus locus coeruleus (LC), the primary source of brain norepinephrine, with its medium-sized dense noradrenergic neurons, is known as the locus coeruleus noradrenergic system (LC-NE) [6]. The LC$\mathrm{NE}$ system affects the neural circuits which form the 
basis of consciousness and cognitive processes. Also, it exhibits modulatory effect on different brain related process such as arousal, sleep-wake cycle, learning, anxiety, pain, mood, and energy metabolism [7]. LC projections innervate various parts of the brain including the cerebellum, spinal cord, and hippocampus (HIPP); on the other hand, it receives inputs from different brain nuclei such as the lateral raphe nucleus and nucleus of the tractus solitarius (NTS) [8]. Due to the substantial connectivity of the LC-NE with several parts of the HIPP, the locus coeruleus plays a crucial role in synaptic amplification of neuronal circuits in this area and thereby on the cognitive functions of the brain such as mood and memory [9].

To better clarify the LC-NA system critical role in different memories, we reviewed several studies and a variety of behavioral tests. Moreover, alongside explaining the critical role of the LC-NE in neuronal circuits, to draw a comprehensive feasible framework to develop future researches, its effect on molecular and cellular processes of memory formation was reviewed.

\section{Methods}

A comprehensive literature search was conducted on PubMed and Scopus databases looking for articles using the following key terms: "Locus coeruleus", "Norepinephrine", "Memory", "Avoidance", "Spatial", "Recognition", "Long-term potentiation", "Neurogenesis", and, "Alzheimer's disease". The key terms were chosen using MeSH and, Boolean operators such as "AND" and "OR" were used to connect the key terms. From May 2021 to July 2021, three researchers conducted the search independently. In this study, papers on learning, memory and cognition associated with the nucleus locus coeruleus were selected, and eventually, 200 articles were included in the study. Next, the articles about Parkinson's disease, depression, as well as those written before 2000, were excluded. Also, to prevent missing valuable studies, the references of the included articles were searched to extract other related studies. Finally, 50 studies were extracted and included in the review.

\section{LC and memory}

3.1 LC and avoidance memory
Avoidance memory is the experience of unpleasant and emotional stimuli that subsequently causes avoidant behaviors to avoid them [10]. Different parts of the brain, such as the amygdala, NTS, and HIPP actually play an important role in the acquisition and consolidation of this memory [11]. More specifically, various studies have shown that different amygdala nuclei have a major role in achieving an avoidance strategy or consolidation of avoidance memory [12]. It has been suggested that LC stimulation can improve avoidance learning and memory as it is the main source of the amygdala norepinephrine [13]. In line with this suggestion, Llorca-Torralba et al. reported, pain as an external stimulus could enhance avoidance memory through the stimulation of LC-NE and intensify its effect on the amygdala [14]. Similarly, according to Fan et al. obtained results of an animal study, corticosterone and stress increase the expression of adrenaline-producing enzymes in LC, boost the function of the basolateral amygdala (BLA) and development of avoidance behavior [15]. Also, Shelkar et al. have been reported that interaction between LC-NE and NO facilitates the expression of avoidance behavior [16]. Moreover, Khakpour-Taleghani et al. revealed that while temporary LC inactivation has no influence on the acquisition of negative experiences during avoidance learning, LC plays a role in the consolidation of this memory and converting short-term into long-term memory [17]. It has also been observed that stimulation of LC noradrenergic nerves increase neuronal firing in the BLA, and triggers anxiety-like responses in rodents [18]. Moreover, it seems that stress can induce a reflexive response in the central region of the amygdala (CeA), thereby potentiates rodents avoidance memory through enhanced BLA neuronal firing following augmentation of tonic LCNE activity (Figure 1) [6].

\subsection{LCand spatial memory}

Spatial memory is the ability to keep encoded and retrieved information which is related to an individual's environment and/or spatial orientation. Indeed, it is a result of the interplay between different cell populations within the HIPP [19], and other various areas of the brain, including the LC. Given the strong mutual neuronal connections between the HIPP and the LC, several studies have been conducted to assay the effect of LC stimulation on the HIPP and 


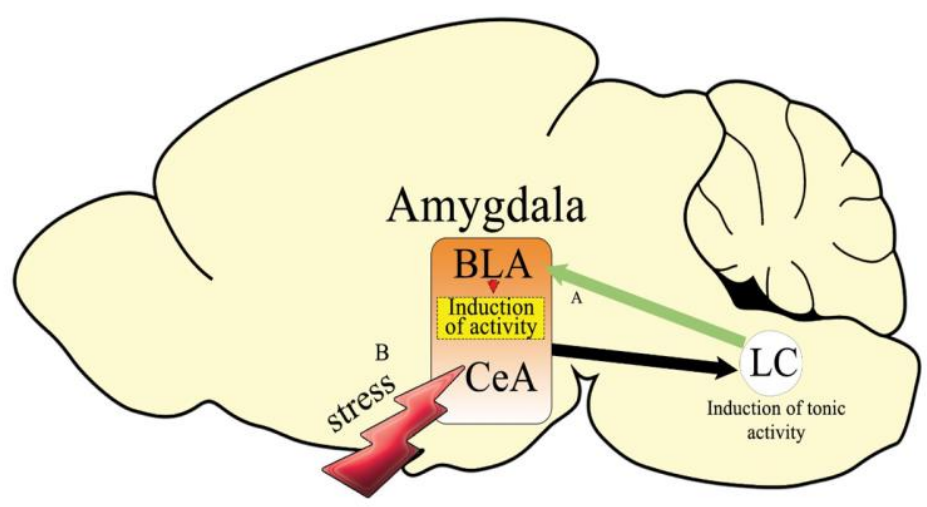

Figure 1. Path A: The amygdala is the main area for avoidance memory formation. The LC noradrenergic projections [green arrow] affect avoidance memory formation in BLA. Path B: Stress, causes the reflective enhanced CeA activity [dark arrow], which in return increases tonic activity of LC-NE and subsequently, the BLA activity increases.

Abbreviations: BLA: basolateral amygdala; CeA: central nucleus of the amygdala; LC: locus coeruleus.

spatial memory. Lemon et al. found that electrical stimulation of the LC could enhance neural encoding of spatial information in hippocampus CA1 region synapses and subsequently resulted in spatial memory improvement [20]. Similarly, in another report, LC stimulation and consequent epinephrine increase enhanced the signal-to-noise ratio of sensory stimuli and thereby could improve spatial memory performance [21]. It should be emphasized that there are some discrepancies on the LC role in spatial memory processing. According to KhakpourTaleghani et al., although the LC-NE activity has an effective role in spatial memory, it is not the only contributing factor in memory formation. Additionally, the bilateral inactivation of LC had no major effect on the spatial memory consolidation [22]. It may be probable that other factors such as the release of norepinephrine from other parts of the brain can mitigate the negative effects of LC destruction on spatial memory consolidation [22]. In contrast to

Figure 2. The brain portions involved in the spatial memory formation. The LC sends noradrenergic (green arrow) and dopaminergic (red arrow) projections to the hippocampus CA1 area and dorsal hippocampus, respectively. Ultimately, these modulatory projections lead to the spatial memory formation.

Abbreviations: LC: locus coeruleus; HIPP: hippocampus; CA1: hippocampal region $\mathrm{CA} 1$; CA3: hippocampal region $\mathrm{CA} 3$; DG: dentate gyrus.
Khakpour-Taleghani and colleagues findings, others have mentioned impaired spatial memory after the destruction of noradrenergic neurons and the associated decrease in norepinephrine level within the brain [23, 24]. It seems, to solve such controversial findings we need further studies to clarify the role of LC-NE in spatial memory formation. Generally, it appears that LC can improve spatial memory through noradrenergic projections and $\beta$-adrenergic receptordependent signaling pathway which has an effect on neurodevelopmental activity in the HIPP [25]. Currently, we know that dopaminergic projections which originate from the LC can improve spatial memory through targeting D1 and D5 receptors across the dorsal hippocampus (Figure 2) [26].

\subsection{LC and recognition memory}

Recognition memory, a hippocampal higher-level function, represents the ability of animals and humans to identify a stimulus or situation which previously had

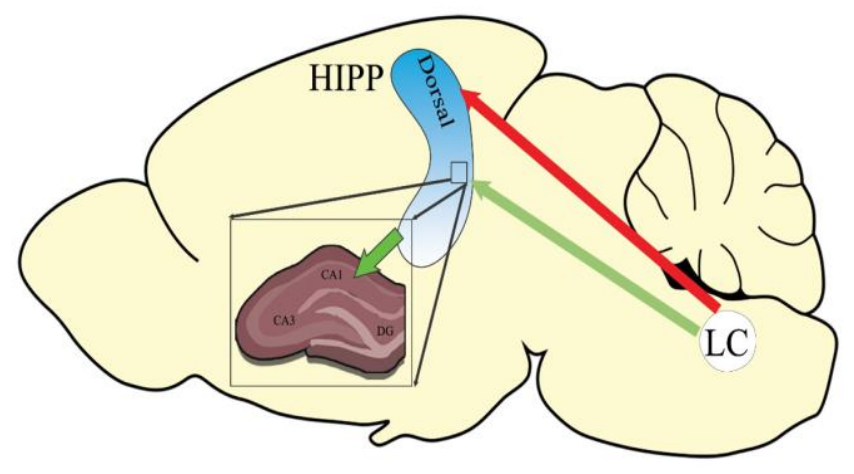


encountered it [27]. Given the robust association between the LC and the HIPP, the hippocampal modulatory effect on recognition memory is not out of scope [28]. Therefore, a large number of studies have been conducted to better understand the relationship between the LC and this type of memory. Titulaer et al. reported that stimulation of LC and subsequent norepinephrine increase in the HIPP ventral area plays a key role in the animals' performance improvement in recognizing new objects [29]. In addition, Kempadoo et al. found that LC light stimulation increases dopamine in dorsal HIPP and eventually improves animal model of recognition memory performance [26]. Similarly, according to Glennon et al. LC stimulation by chemical or even external stimuli such as hearing can also improve recognition memory [30]. In addition to the bilateral relationship between LC and HIPP, according to Mello-Carpes et al. findings, the LC-HIPP-NTSparagigantocellularis (PGi) pathway can significantly affect recognition memory performance [31]. Furthermore, the frontal cortex-LC pathway is another modulator of this memory through $\alpha_{2}$ adrenoceptor signaling [32]. Based on the abovementioned reports, it can be concluded that the neurological activity of locus coeruleus and its noradrenergic projections to different brain areas, like the HIPP, play a pivotal role in the development of this higher function memory (Figure 3) [29].

\section{LC and CNS neurological changes}

\subsection{LC and neurogenesis}

Neurogenesis is a process in the central nervous system (CNS) of adult vertebrates to produce more nerve cells [33]. Among the various factors, the LC-NE system has a major role in the neurogenesis of the CNS at different stages of life and in a variety of ways [34].

Figure 3. Path A. The LC dopaminergic projections (red arrow) to the dorsal hippocampus have a significant role in development of recognition memory. Path B. LC-NE (green arrow) promotes the recognition memory by its effect on the ventral hippocampus. Path C. The LC-HIPP-NTS-PGi pathway (dark arrow) has an effective role in recognition memory function. Path D. LC-NE (green arrow) inhibitory effect on the pre-frontal cortex modulates recognition memory performance.

Abbreviations: LC: locus coeruleus; HIPP: hippocampus; PFC: prefrontal cortex; NTS: nucleus of the solitary tract; PGi: paragigantocellularis.
It has been observed that LC-NE induced neurogenesis begins shortly before birth and reaches its peak in infancy. It exerts significant beneficial effects on learning and memory by its trophic effect on the CNS [35]. Also, the LC has an improving role in the neurogenesis of other brain nuclei. According to Guérin et al. study, LC removal in transgenic immature mice leads to the newborn cells death and the polysialylated neural cell adhesion molecule [PSANCAM] decrease in the olfactory bulb and eventually, olfactory memory reduction [34]. Also, Bortolotto et al. found that the LC-NE intensifies HIPP nerveproducing cells (NPCs) neurogenesis through $\beta_{2}-$ adrenoceptor stimulation [36]. Furthermore, Fan et al. reported that alterations of $\mathrm{LC}$ cells gene expression can influence neurogenesis of HIPP. They revealed that in older mice the expression of a certain type of LC cells transcription factor could enhance cell division in animals' dentate gyrus region [37]. Also, it has been observed that genetic alterations such as LC cell's nucleus miRNA increase, exert trophic effect on the HIPP [38]. Moreover, according to Coradazzi and colleagues research, destruction of the LC, directly diminishes neurogenesis and reduces reproductive cells of the HIPP [39]. Finally, it should be noted that in addition to the LC-NE positive effect on neurogenesis, it also exerts neuroprotective effect through multiple mechanisms including decreasing the oxidative stress and inflammatory responses as well as enhancing endotrophic-neurotrophic systems (NTFs), especially brain-derived neurotrophic factor (BDNF), neurotransmitter production, cholinergic cell survival and also, modulation of CNS energy metabolism alongside with regulation and maintain extracellular homeostasis [40].

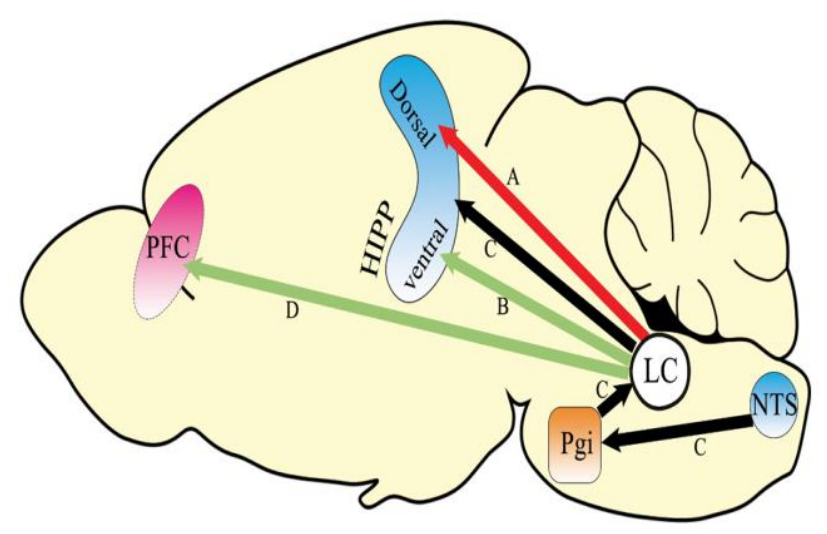




\subsection{LC and neural plasticity}

Long-term potentiation (LTP) is a form of neuroplasticity that is thought to be a common cellular mechanism for many learning and memory processes [41]. The LC-NE system plays a pivotal role in the development of LTP in important memory processing centers such as the dentate gyrus and the CA1 region of the hippocampus. NE exerts its positive effect through different members of the adrenoreceptor family [42]. Quinlan et al. found that in animal models, the LC stimulation with light can lead to LTP formation in the dentate gyrus and subsequent longterm learning and memory improvement [43]. According to Reid et al. following simultaneous stimulation of the perforant pathway and the LC, norepinephrine release starts and results in hippocampal neuronal plasticity and the initiation of learning process [44]. Also, Lashgari et al. has reported that unlike short periods, over a long period of the time, LC noradrenergic system can induce LTP through protein synthesis enhancement [45]. Moreover, according to Hammerschmidt et al. results, the LC destruction can decrease the LTP occurrence in the HIPP through the reduction of CaMKII and NR2A and finally leads to memory loss [46]. In addition, according to Tamano et al. report, through $\beta$ adrenoreceptors activation the LC-NE regulates $\mathrm{Zn}^{2+}$ ion concentration and subsequently beta-amyloid decrement can facilitate the hippocampal dentate gyrus LTP production [47]. It should be noted that the sensitivity towards epinephrine is not the same at different parts of the HIPP, and each region expresses a certain level of LTP, depending on its $\beta$ adrenoreceptors expression [48]. Titulaer et al. found that in addition to $\mathrm{NE}$, the nucleus locus coeruleus dopaminergic projections have an effective role in the LTP production in the ventral HIPP which is involved in recognition memory. They suggested that it acts through stimulation of hippocampal D1/5 receptors [29].

\section{LC and Alzheimer's disease}

Many studies have demonstrated that LC is highly susceptible to various toxins and infections, and in many cases, is the primary site where $\mathrm{AD}$ pathology occurs [49]. German et al. found that amyloid-beta build-up in the LC results in the destruction of its neurons [50]. Similarly, Ross et al. observed that excessive activity of the $\mathrm{LC}$ in response to hormonal stressors such as the corticotropin-releasing factor may cause production and deposition of amyloid-beta and introduced it as one of the most important pathologies of Alzheimer's disease (AD) [51]. Aghajanov et al. reported that in animal models of Alzheimer's disease, elevated level of oxidative stress, together with increased concentration of monoamines, particularly norepinephrin, is accompanied by severe structural damage to LC monoaminergic neurons [52]. Also, Hopp et al. found that neuroinflammation can induce $\mathrm{AD}$ symptoms by disrupting the regulation of calcium ions in the $\mathrm{LC}$ and leads to impaired nervous pacemaker activity [53]. Furthermore, it has been observed that loss of the LC noradrenergic neurons or its decreased activity causes $\mathrm{AD}$ symptoms in different parts of the brain [54, 55]. For example, Kelly et al. demonstrated that degeneration of the LC-NE system increases the risk of early $\mathrm{AD}$ through impairing vascular and neurological function in the brain areas associated with memory and cognitive performances [55]. Another hypothesis is that the primary signaling of aberrant forms of tau protein begins in the LC [56]. Kang et al. have been reported that aberrant forms of tau protein not only cause neurological dysfunction but also are capable of spreading memory processing regions of the brain and might contribute to the $\mathrm{AD}$ development [57]. Moreover, we know that increased LC activity by neurotrophic factors, may have beneficial effects in preventing neuropsychological signs of $\mathrm{AD}$ [58].

\section{Conclusion}

The nucleus locus coeruleus through its noradrenergic projections affects various parts of memory processing areas of the brain. Also, LC-NE as a potent modulatory system, as well as other important memory processing cholinergic and dopaminergic systems, play an important role in memory formation. In addition, through the stimulant noradrenergic projections to the baso-lateral amygdala, LC has an effective role in enhancing avoidance memory. Of course, the effective role of LC in other brain areas such as NTS should be considered in the avoidance memory formation. It develops spatial memory mainly through $\beta$-adrenergic signaling in the CA1 region of the hippocampus. Furthermore, due to its stimulatory effect on the LCHIPP-NTS- PGi pathway, especially the hippocampus and the modulatory effect on the frontal 
cortex, LC has a pivotal role in the development of cognitive memory. On the other hand, LC-NE can lead to permanent LTP production and neurogenesis through the induction of cellular level changes in gene expression, protein synthesis, and ions concentration in nuclei and/or circuits involved in memory formation. Moreover, we must emphasize that the role of the LC dopaminergic projections should not be overlooked in the memory development. Based on the previous evidences, injuries, degeneration or destruction of the LC and LC-NE system may also contribute to memory loss and neurodegenerative diseases such as Alzheimer's disease. Although, this issue needs more extensive research to prove, this finding can be considered as a clear perspective for the development of treatment planning platforms based on the strengthening of the LC-NE system.

\section{Author contributions}

BKH: provided main title of study, studies platform, collection of data, and drafting. MAH: provided collection of data, draw figures, and drafting. AJ: provided scientific review, critical revisions, and native English edition.

\section{Conflict of interests}

The authors declare that they have no conflict of interest.

\section{Ethical declarations \\ Not applicable.}

\section{Financial Support}

This research did not receive any specific grant from funding agencies in the public, commercial, or not-for-profit sectors.

\section{References}

1. Anderson JR. Learning and memory: An integrated approach: John Wiley \& Sons Inc; 2000.

2. Ben-Yakov A, Dudai Y, Mayford MR. Memory Retrieval in Mice and Men. Cold Spring Harb Perspect Biol. 2015; 7(12):a021790.

3. Morris R, Tarassenko L. Cognitive Systems Remember Experience: Introduction. In: Morris R, Tarassenko L, Kenward M, editors. Cognitive Systems - Information Processing Meets Brain Science. London: Academic Press; 2006. p. 191-2.

4. Sara SJ. Locus Coeruleus in time with the making of memories. Curr Opin Neurobiol. 2015; 35:87-94.

5. Fukabori R, Iguchi Y, Kato S, Takahashi K, Eifuku S, Tsuji S, et al. Enhanced Retrieval of Taste Associative Memory by
Chemogenetic Activation of Locus Coeruleus Norepinephrine Neurons. J Neurosci. 2020; 4O(43):8367-85.

6. James T, Kula B, Choi S, Khan SS, Bekar LK, Smith NA. Locus coeruleus in memory formation and Alzheimer's disease. Eur $\mathrm{J}$ Neurosci. 2020; 00:1- 12.

7. Duszkiewicz AJ, McNamara CG, Takeuchi T, Genzel L. Novelty and Dopaminergic Modulation of Memory Persistence: A Tale of Two Systems. Trends Neurosci. 2019; 42(2):102-14.

8. Sara SJ, Bouret S. Orienting and reorienting: the locus coeruleus mediates cognition through arousal. Neuron. 2012; 76(1):130-41.

9. Loughlin SE, Foote SL, Bloom FE. Efferent projections of nucleus locus coeruleus: topographic organization of cells of origin demonstrated by three-dimensional reconstruction. Neuroscience. 1986; 18(2):291-306.

10. Falibene A, Roces F, Rössler W. Long-term avoidance memory formation is associated with a transient increase in mushroom body synaptic complexes in leaf-cutting ants. Front Behav Neurosci. 2015; 9:84.

11. García-Medina NE, Miranda MI. Nucleus of the solitary tract chemical stimulation induces extracellular norepinephrine release in the lateral and basolateral amygdala. Brain Stimul. 2013; 6(2):198-201.

12. Segura-Torres P, Aldavert-Vera L, Gatell-Segura A, RedolarRipoll D, Morgado-Bernal I. Intracranial self-stimulation recovers learning and memory capacity in basolateral amygdala-damaged rats. Neurobiol Learn Mem. 2010; 93(1):117-26.

13. McCall JG, Siuda ER, Bhatti DL. Locus coeruleus to basolateral amygdala noradrenergic projections promote anxiety-like behavior. Elife. 2017; 6:e18247.

14. Llorca-Torralba M, Suárez-Pereira I, Bravo L, CamarenaDelgado C, Garcia-Partida JA, Mico JA, et al. Chemogenetic Silencing of the Locus Coeruleus-Basolateral Amygdala Pathway Abolishes Pain-Induced Anxiety and Enhanced Aversive Learning in Rats. Biol Psychiatry. 2019; 85(12):1021-35.

15. Fan Y, Chen P, Li Y, Cui K, Noel DM, Cummins ED, et al. Corticosterone administration up-regulated expression of norepinephrine transporter and dopamine $\beta$-hydroxylase in rat locus coeruleus and its terminal regions. J Neurochem. 2014; 128(3):445-58.

16. Shelkar GP, Gakare SG, Chakraborty S, Dravid SM, Ugale RR. Interactions of nitric oxide with $\alpha 2$-adrenoceptors within the locus coeruleus underlie the facilitation of inhibitory avoidance memory by agmatine. Br J Pharmacol. 2016; 173(17):2589-99.

17. Khakpour-Taleghani B, Lashgari R, Aavani T, Haghparast A, Naderi N, Motamedi F. The locus coeruleus involves in consolidation and memory retrieval, but not in acquisition of inhibitory avoidance learning task. Behav Brain Res. 2008; 189(2):257-62.

18. Crone JS, Schurz M, Höller Y, Bergmann J, Monti M, Schmid $\mathrm{E}$, et al. Impaired consciousness is linked to changes in effective connectivity of the posterior cingulate cortex within the default mode network. Neuroimage. 2015; 110:101-9.

19. Baram TZ, Donato F, Holmes GL. Construction and disruption of spatial memory networks during development. Learn Mem. 2019; 26(7):206-18.

20. Lemon N, Aydin-Abidin S, Funke K, Manahan-Vaughan D. Locus coeruleus activation facilitates memory encoding and induces hippocampal LTD that depends on beta-adrenergic receptor activation. Cereb Cortex. 2009; 19(12):2827-37. 
21. Collier TJ, Greene JG, Felten DL, Stevens SY, Collier KS. Reduced cortical noradrenergic neurotransmission is associated with increased neophobia and impaired spatial memory in aged rats. Neurobiol Aging. 2004; 25(2):209-21.

22. Khakpour-Taleghani B, Lashgari R, Motamedi F, Naghdi N. Effect of reversible inactivation of locus ceruleus on spatial reference and working memory. Neuroscience. 2009; 158(4):1284-91.

23. Choudhary P, Pacholko AG, Palaschuk J, Bekar LK. The locus coeruleus neurotoxin, DSP4, and/or a high sugar diet induce behavioral and biochemical alterations in wild-type mice consistent with Alzheimers related pathology. Metab Brain Dis. 2018; 33(5):1563-71.

24. Litteljohn D, Nelson E, Hayley S. IFN- $\gamma$ differentially modulates memory-related processes under basal and chronic stressor conditions. Front Cell Neurosci. 2014; 8:391.

25. Hansen N, Manahan-Vaughan D. Locus Coeruleus Stimulation Facilitates Long-Term Depression in the Dentate Gyrus That Requires Activation of $\beta$-Adrenergic Receptors. Cereb Cortex. 2015; 25(7):1889-96.

26. Kempadoo KA, Mosharov EV, Choi SJ, Sulzer D, Kandel ER. Dopamine release from the locus coeruleus to the dorsal hippocampus promotes spatial learning and memory. Proc Natl Acad Sci U S A. 2016; 113(51):14835-40.

27. Moreno-Castilla P, Guzman-Ramos K, Bermudez-Rattoni F. Chapter 28 - Object Recognition and Object Location Recognition Memory - The Role of Dopamine and Noradrenaline. In: Ennaceur A, de Souza Silva MA, editors. Handb Behav Neurosci: Elsevier; 2018. p. 403-13.

28. Evans AK, Park HH, Saw NL, Singhal K, Ogawa G, Leib RD, et al. Age-related neuroinflammation and pathology in the locus coeruleus and hippocampus: beta-adrenergic antagonists exacerbate impairment of learning and memory in aged mice. Neurobiol Aging. 2021; 106:241-56.

29. Titulaer J, Björkholm C, Feltmann K, Malmlöf T, Mishra D, Bengtsson Gonzales $\mathrm{C}$, et al. The Importance of Ventral Hippocampal Dopamine and Norepinephrine in Recognition Memory. Front Behav Neurosci. 2021; 15:667244.

30. Glennon E, Carcea I, Martins ARO, Multani J, Shehu I, Svirsky MA, et al. Locus coeruleus activation accelerates perceptual learning. Brain Res. 2019; 1709:39-49.

31. Mello-Carpes PB, Izquierdo I. The Nucleus of the Solitary Tract $\rightarrow$ Nucleus Paragigantocellularis $\rightarrow$ Locus Coeruleus $\rightarrow$ CA1 region of dorsal hippocampus pathway is important for consolidation of object recognition memory. Neurobiol Learn Mem. 2013; 100:56-63.

32. Wagner-Altendorf TA, Fischer B, Roeper J. Axonal projectionspecific differences in somatodendritic $\alpha 2$ autoreceptor function in locus coeruleus neurons. Eur J Neurosci. 2019; 5O(11):3772-85.

33. Engler A, Zhang R, TaylorV. Notch and Neurogenesis. Adv Exp Med Biol. 2018; 1066:223-34.

34. Guérin D, Sacquet J, Mandairon N, Jourdan F, Didier A. Early locus coeruleus degeneration and olfactory dysfunctions in Tg2576 mice. Neurobiol Aging. 2009; 30(2):272-83.

35. Duarte EC, de Azevedo MS, Pereira GA, Fernandes MD, Lucion $\mathrm{AB}$. Intervention with the mother-infant relationship reduces cell proliferation in the Locus Coeruleus of female rat pups. Behav Neurosci. 2017; 131(1):83-91.
36. Bortolotto V, Bondi H, Cuccurazzu B, Rinaldi M, Canonico PL, Grilli M. Salmeterol, a $\beta 2$ Adrenergic Agonist, Promotes Adult Hippocampal Neurogenesis in a Region-Specific Manner. Front Pharmacol. 2019; 10:1000.

37. Fan Y, Zeng F, Brown RW, Price JB, Jones TC, Zhu MY. Transcription Factors Phox2a/2b Upregulate Expression of Noradrenergic and Dopaminergic Phenotypes in Aged Rat Brains Neurotox Res. 2020; 38(3):793-807.

38. Launay JM, Mouillet-Richard S, Baudry A, Pietri M, Kellermann O. Raphe-mediated signals control the hippocampal response to SRI antidepressants via miR-16. Transl Psychiatry. 2011; 1(11):e56.

39. Coradazzi M, Gulino R, Fieramosca F, Falzacappa LV, Riggi M, Leanza G. Selective noradrenaline depletion impairs working memory and hippocampal neurogenesis. Neurobiol Aging. 2016; 48:93-102.

40. Xu W, Yu JT, Tan MS, Tan L. Cognitive reserve and Alzheimer's disease. Mol Neurobiol. 2015; 51(1):187-208.

41. Bergado JA, Frey S, López J, Almaguer-Melian W, Frey JU. Cholinergic afferents to the locus coeruleus and noradrenergic afferents to the medial septum mediate LTP-reinforcement in the dentate gyrus by stimulation of the amygdala. Neurobiol Learn Mem. 2007; 88(3):331-41.

42. Hansen N, Manahan-Vaughan D. Hippocampal long-term potentiation that is elicited by perforant path stimulation or that occurs in conjunction with spatial learning is tightly controlled by beta-adrenoreceptors and the locus coeruleus. Hippocampus. 2015; 25(11):1285-98.

43. Quinlan MAL, Strong VM, Skinner DM, Martin GM, Harley CW, Walling SG. Locus Coeruleus Optogenetic Light Activation Induces Long-Term Potentiation of Perforant Path Population Spike Amplitude in Rat Dentate Gyrus. Front Syst Neurosci. 2018; 12:67.

44. Reid AT, Harley CW. An associativity requirement for locus coeruleus-induced long-term potentiation in the dentate gyrus of the urethane-anesthetized rat. Exp Brain Res. 2010; 200(2):151-9. 45. Lashgari R, Khakpour-Taleghani B, Motamedi F, Shahidi S. Effects of reversible inactivation of locus coeruleus on long-term potentiation in perforant path-DG synapses in rats. Neurobiol Learn Mem. 2008; 90(2):309-16.

46. Hammerschmidt T, Kummer MP, Terwel D, Martinez A, Gorji A, Pape HC, et al. Selective loss of noradrenaline exacerbates early cognitive dysfunction and synaptic deficits in APP/PS1 mice. Biol Psychiatry. 2013; 73(5):454-63.

47. Tamano H, Ishikawa Y, Shioya A, Itoh R, Oneta N, Shimaya R, et al. Adrenergic $\beta$ receptor activation reduces amyloid $\beta(1-42)-$ mediated intracellular $\mathrm{Zn}(2+)$ toxicity in dentate granule cells followed by rescuing impairment of dentate gyrus LTP. Neurotoxicology. 2020; 79:177-83.

48. Hagena H, Hansen N, Manahan-Vaughan D. $\beta$-Adrenergic Control of Hippocampal Function: Subserving the Choreography of Synaptic Information Storage and Memory. Cereb Cortex. 2016; 26(4):1349-64.

49. Mather M, Harley CW. The Locus Coeruleus: Essential for Maintaining Cognitive Function and the Aging Brain. Trends Cogn Sci. 2016; 20(3):214-26.

50. German DC, Nelson O, Liang F, Liang CL, Games D. The PDAPP mouse model of Alzheimer's disease: locus coeruleus neuronal shrinkage. J Comp Neurol. 2005; 492(4):469-76. 
51. Ross JA, Alexis R, Reyes BAS, Risbrough V, Van Bockstaele EJ. Localization of amyloid beta peptides to locus coeruleus and medial prefrontal cortex in corticotropin releasing factor overexpressing male and female mice. Brain Struct Funct. 2019; 224(7):2385-405.

52. Aghajanov M, Chavushyan V, Matinyan S, Danielyan M, Yenkoyan K. Alzheimer's disease-like pathology-triggered oxidative stress, alterations in monoamines levels, and structural damage of locus coeruleus neurons are partially recovered by a mix of proteoglycans of embryonic genesis. Neurochem Int. 2019; 131:104531.

53. Hopp SC, Royer SE, D'Angelo HM, Kaercher RM, Fisher DA, Wenk GL. Differential neuroprotective and anti-inflammatory effects of L-type voltage dependent calcium channel and ryanodine receptor antagonists in the substantia nigra and locus coeruleus. $J$ Neuroimmune Pharmacol. 2015; 10(1):35-44.

54. Olivieri P, Lagarde J, Lehericy S, Valabrègue R, Michel A, Macé $\mathrm{P}$, et al. Early alteration of the locus coeruleus in phenotypic variants of Alzheimer's disease. Ann Clin Transl Neurol. 2019; 6(7):1345-51.

55. Kelly SC, McKay EC, Beck JS, Collier TJ, Dorrance AM, Counts SE. Locus Coeruleus Degeneration Induces Forebrain Vascular Pathology in a Transgenic Rat Model of Alzheimer's Disease. J Alzheimers Dis. 2019; 70(2):371-88.

56. Kaufman SK, Del Tredici K, Thomas TL, Braak H, Diamond MI. Tau seeding activity begins in the transentorhinal/entorhinal regions and anticipates phospho-tau pathology in Alzheimer's disease and PART. Acta Neuropathol. 2018; 136(1):57-67.

57. Kang SS, Liu X, Ahn EH, Xiang J, Manfredsson FP, Yang X, et al. Norepinephrine metabolite DOPEGAL activates AEP and pathological Tau aggregation in locus coeruleus. J Clin Invest. 2020; 130(1):422-37.

58. Braun D, Feinstein DL. The locus coeruleus neuroprotective drug vindeburnol normalizes behavior in the $5 \mathrm{xFAD}$ transgenic mouse model of Alzheimer's disease. Brain Res. 2019; 1702:29-37. 